\title{
Keck Interferometer autoaligner: algorithms and techniques
}

\section{Michael A. Hrynevych, Kevin Tsubota, Robert F. Smythe, Wayne Dahl, James Bell, et al.}

Michael A. Hrynevych, Kevin Tsubota, Robert F. Smythe, Wayne Dahl, James Bell, M. Mark Colavita, John Gathright, Forrest Meggars, Christopher R. Neyman, Andy C. Rudeen, Gerard Theodore van Belle, Peter L.

Wizinowich, "Keck Interferometer autoaligner: algorithms and techniques," Proc. SPIE 5491, New Frontiers in Stellar Interferometry, (20 October 2004); doi: $10.1117 / 12.552417$

Event: SPIE Astronomical Telescopes + Instrumentation, 2004, Glasgow, United Kingdom 


\title{
Keck Interferometer autoaligner: algorithms and techniques
}

\author{
Michael A. Hrynevych ${ }^{* a}$, Kevin Tsubota $^{a}$, Robert Smythe ${ }^{\mathrm{b}}$, Wayne Dahl ${ }^{\mathrm{a}}$, Jim Bell $^{\mathrm{a}}$, M. Mark \\ Colavita $^{\mathrm{b}}$, John Gathright ${ }^{\mathrm{a}}$, Forest Meggars ${ }^{\mathrm{a}}$, Christoper Neyman ${ }^{\mathrm{a}}$, Andy Rudeen ${ }^{\mathrm{a}}$, Gerard van \\ Belle $^{\mathrm{c}}$, Peter Wizinowich ${ }^{\mathrm{a}}$ \\ ${ }^{a}$ W. M. Keck Observatory, 65-1120 Mamalahoa Hwy, Kamuela, HI, USA 96743 \\ ${ }^{\mathrm{b} J e t}$ Propulsion Laboratory, California Institute of Technology, 4800 Oak Grove Drive, \\ Pasadena, CA, USA 91109 \\ ${ }^{c}$ Michelson Science Center, California Institute of Technology, 770 So. Wilson Ave., \\ Pasadena, CA, USA 91125
}

\begin{abstract}
The Keck Interferometer includes an autoalignment system consisting of pop-up targets located at strategic locations along the beam trains of each arm of the instrument along with a sensor and control system. We briefly describe the hardware of the system and then proceed to a description of the two operational modes of the system. These are: 1) to provide an initial alignment of the coudé paths in each arm, and 2) to recover coudé alignments between changes of the static delay sled positions. For the initial alignment mode, we review the system performance requirements along with the software used for image acquisition and centroiding. For coudé alignment recovery, we describe beam-train surveys through the static delay (Long Delay Line) and criteria for a successful recovery of a coudé alignment. Finally, we describe the results of testing of the autoalignment system.
\end{abstract}

Keywords: Interferometry, alignment, automation, beamtrain, Keck Interferometer

\section{BACKGROUND}

\subsection{Introduction}

Starlight entering the Keck Interferometer, after emerging from each of the respective Adaptive Optics (AO) systems, has some 19 reflective elements to negotiate in each input arm before making its way onto the beam-combining table. After this there are several more reflections before it is finally combined, interfered, and focused into the fiber inputs of the fringe detector/tracker. The mirrors involved make up elements of: phase delay systems, beam compressors, and simple beam transport. In particular, phase delay in the interferometer is achieved using two delay line systems: the static delay is provided by the Long Delay Lines (LDL), these are stationary during fringe detection; and the dynamic delay system, the Fast Delay Lines (FDL) that track delay during observation.

The number of mirrors involved in this current mode of operation (the visibility squared mode ${ }^{1}$, the first of the four major modes of the Keck Interferometer) already makes a formidable case for the implementation of an automated beam train alignment system. Other modes will introduce additional beam lines; for example, the Nulling interferometer will add secondary beam trains along side the existing primary beam trains. Hence, for the present and future modes of the interferometer, auto alignment offers a variety of advantages over manual alignment, which include:

- Autoalignment is potentially more quickly accomplished than manual alignment. This time consideration is not only a factor in the preparations for a night's observation, but also critical in reconfiguring the LDL position in

\footnotetext{
* mhrynevych@keck.hawaii.edu; phone 808 885-7887; fax 808 881-3535; http://www.keckobservatory.org; W. M. Keck Observatory, 65-1120 Mamalahoa Hwy, Kamuela, HI 96743
} 
the course of a night's observation. This enables quick changes between different observing programs and/or for viewing widely separated portions of sky during the night.

- Autoalignment is potentially of much greater precision and repeatability than manual alignment.

- Some beam train mirrors, particularly those along the coudé train, are difficult to access.

- Disturbances of the laboratory environment are substantially less performing alignments via remote actuation.

- Reduction of the amount of resources (personnel and time) required to prepare for interferometric observations.

Elements of this system have been described previously; that description focused on the details of the hardware and the driver software for the autoaligner ${ }^{2}$. In this paper we present an overview of the beam train in the Keck Interferometer, the manual procedure to perform the necessary alignments, the hardware and software to perform an autoalignment, and report on the current performance of beam train autoalignment system.

\subsection{The beamtrain}

An overview of the beam path in one of the arms of the Keck Interferometer can be gleaned from Fig. 1. Here, the segmented mirror of Keck 1 is shown at left with starlight entering the telescope from overhead and exiting into the AO system. On leaving the AO system, starlight encounters the first of the interferometer beam train mirrors at M2 located on the Dual Star Module (DSM) on the Nasmyth platform. At this point the starlight is a collimated 4" beam. Starlight encounters an additional two mirrors (M3 and M4) on the DSM before leaving the Nasmyth platform toward the coudé tunnel. Both M5 and M6 are on the telescope structure, M6 being the final point of contact that the light beam has with the rotating structure of the telescope.

From M7 and M8 starlight passes through the coudé tunnel and onward over the top of the LDL area, toward M9, located in the switchyard area. Mirrors M9 and M10 steer light to the appropriate LDL sled. The LDL provides the static delay for an observation and provides up to $70 \mathrm{~m}$ of optical path delay for a particular interferometer arm. The LDL sled holds the M11, a flat mirror that directs light toward M14. It may be noted that there are two "missing" mirrors (M12 and M13; not shown) in the sequence that are associated with a potential future upgrade that would allow the LDL to introduce even more optical delay - essentially double the potential delay length.

From M14, starlight is directed toward the beam train's FDL cart, passing beneath the Beam Reducing Telescope (BRT). The FDL cart is a cats-eye reflector comprised of a large concave mirror and a small PZT-mounted flat mirror. Starlight is initially incident on the lower portion of the concave mirror that brings it to focus at the flat mirror location before being reflected toward the upper surface of the concave mirror where it is re-collimated and sent out of the FDL cart. The FDL has the capacity to introduce up to $15 \mathrm{~m}$ of optical delay and is used to track delay during observations. From the FDL, starlight enters the BRT, comprised of a pair of powered optics, which reduces beam diameter from 4" to $1 "$.

In this 1" space, starlight now makes its way toward the detectors; a dichroic picks off light for the fringe tracker on the beam-combining table; another picks off light for the angle tracker and the visible remnants find themselves at the autoalignment camera (AACAM).

The interferometer's Keck 2 beam train is similar, entering the diagram in Fig. 1 from the right and passing through its own set of M9 and M10 mirrors into the LDL. Thereafter, it essentially traces a path alongside the Keck 1 beam train all the way to the fringe detector and angle trackers.

Of this entire beam train, the autoalignment system deals with alignments of mirrors in the segment from M4 through to M14. Of particular interest is the alignment through the portion that passes from the rotating Keck telescope structure to the stationary mirrors below, the segment between M6 and M7. For observations to proceed unimpeded with an unvignetted beam line, the M6 and M7 segment must not only be aligned to the mirrors of the rest of the beamtrain but also to the azimuthal mechanical rotation axis of the telescope. It is an essential ability to rapidly and efficiently reach this alignment both in the initial setup for nightly observing and after reconfigurations of the LDL sleds during the night for realizing the Keck Interferometer's full functionality. The central topic of this paper is to describe how this alignment is obtained, the strategy that has been used to automate it, and report on progress to date of achieving this goal. 


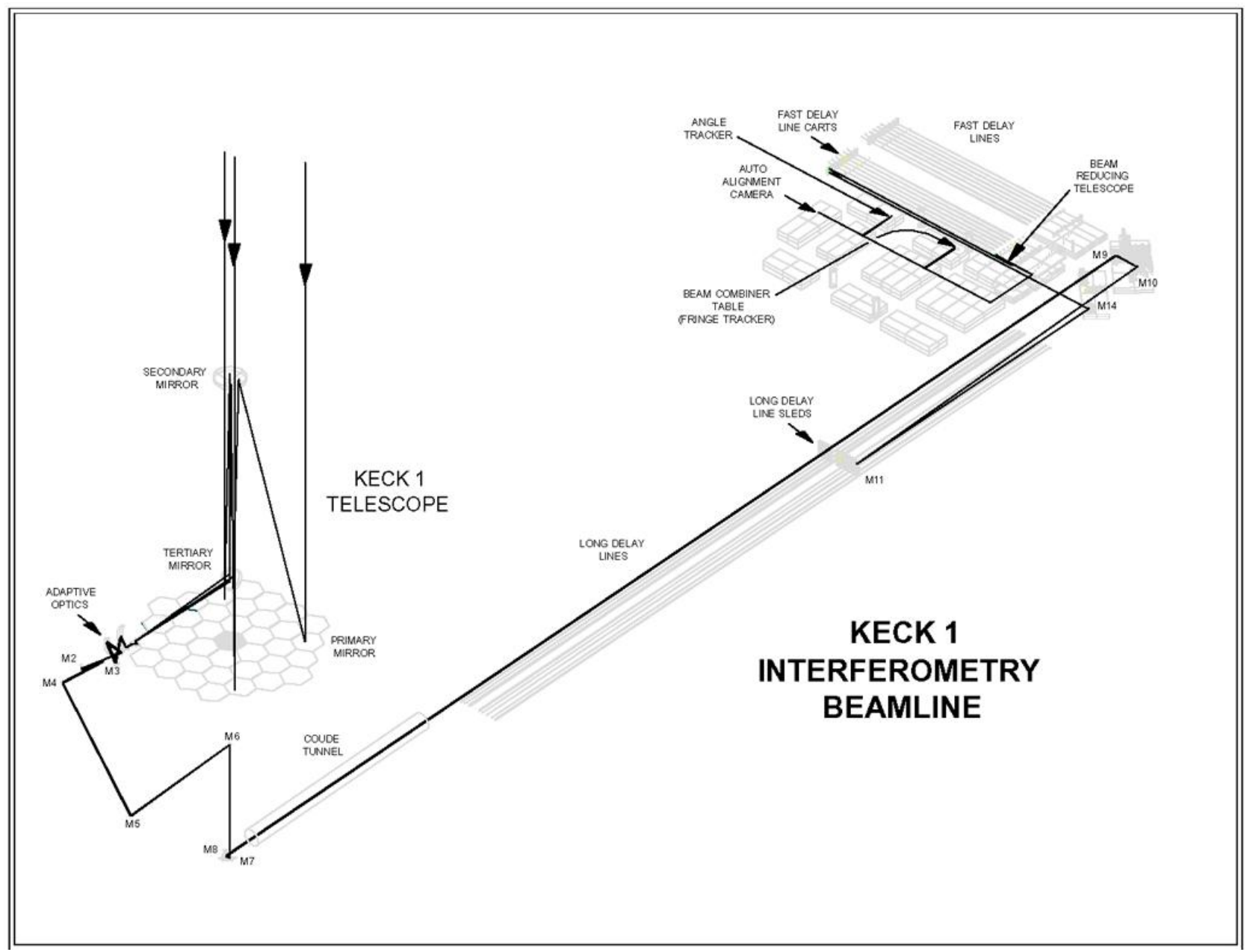

Fig. 1. The Keck Interferometer beam train from Keck 1, from telescope to fringe tracker. Keck 2 has a similar input entering from the right.

\subsection{Elements of automation: the actuators and targets}

Elements of the autoalignment system hardware are located along many points of the beam train in the form of mirror actuators and alignment targets. The autoalignment camera (AACAM) at the end of the beam train completes the inventory of the autoalignment beam train hardware.

The mirror actuators are a commercially available product - the Newport 850G DC servo long travel motors that provide up to $50 \mathrm{~mm}$ of motion. At the actuated mirrors, they are used as pairs for $\mathrm{x}$ and $\mathrm{y}$ actuation and are directly mounted to the mirror mounts. They are under software control through EPICS (Experimental Physics Industrial Control Software), a distributed control system software package, which runs within the VxWorks real-time operating system.

The LED alignment targets are retractable LEDs that are mounted on a swing arm that allows the illuminated target to be swung into place and retracted with the LED off, as required. These are custom-built devices. They are installed so that the extended LED target is at the location of a well-aligned coudé beam train. It is assumed that the LEDs are positioned repeatedly and, thus, define a fiduical beam alignment. These are designated according to the proximity of the nearest actuated mirror (e.g., the target near M6 is L6). These too are EPICS-controlled devices. 

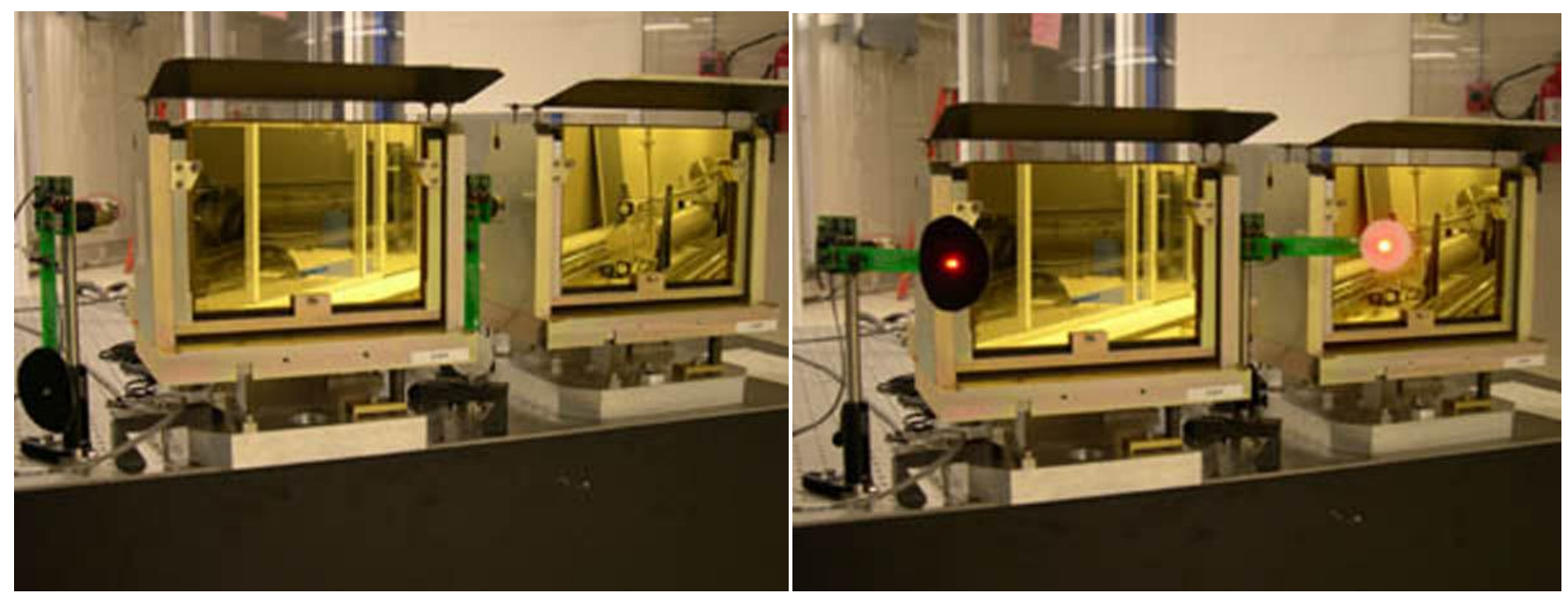

Fig. 2: The LED pop-up targets in front of the M14 mirrors. At left they are retracted and out of the beam line. At right they are in their actuated mode, in position and illuminated.

Table 1 summarizes the location of the actuation elements and LED target locations. Generally, where there are two nearby mirrors (less than a few meters) only one of these has been actuated and the associated LED targets are typically located close to these actuated mirrors. The LED target on the Nasmyth deck, L4, is the exception here, as it is the endpoint of alignments for the autoalignment system.

The AACAM is an inexpensive CCD camera set behind an iris and singlet lens without any focus adjustment. This optical system, with its relatively slow focus (f/40), has a large depth of focus and is still able to observe both near and distant LED targets. As many of these targets will be out of focus images on the camera, a concern in the design was obtaining a sufficient intensity for the out-of-focus images while maintaining the simplicity of the fixed focus. The fixed focus also guarantees that the optical axis remains constant irrespective of which LED target is observed.

The output video frames of AACAM can be captured with a framegrabber where the images are digitized and centroids of LED targets are derived. These coordinates are the feedback for performing autoalignments. The effective usable area of the frame-grabber's image is approximately 200 x 200 pixels.

Table 1: Inventory and location of actuators and targets along each arm of the interferometer beam train as part of the autoalignment system hardware.

\begin{tabular}{|c|c|c|}
\hline Mirror Location & Actuation & LED Targets \\
\hline M4 & None & L4 \\
\hline M5 & None & L6 \\
\hline M6 & $850 \mathrm{G}$ & L7 \\
\hline M7 & $850 \mathrm{G}$ & L9 \\
\hline M8 & None & \\
\hline M9 & $850 \mathrm{G}$ & L11 \\
\hline M10 & None & L14 \\
\hline M11 & $850 \mathrm{G}$ & \\
\hline M14 & $850 \mathrm{G}$ & \\
\hline
\end{tabular}




\section{BEAM TRAIN ALIGNMENTS}

\subsection{Preliminaries}

Initial beam train alignment is performed in the opposite direction to the direction of starlight. A collimated boresight laser that is launched from the beam-combining table is used to make the alignment. This is produced as a 1 " collimated beam, but typically is stopped to $1 / 4^{\prime \prime}$ to provide a pencil beam that is useful for alignments. A collimated white light beam is also produced on the beam-combining table and is used in the alignments of the angle tracker and fringe tracker, as well as in the production of internal fringes. It is co-aligned to the boresight laser. Both AO systems have white light sources to simulate starlight that are also employed in the overall alignment procedure.

\subsection{The coudé alignment}

For each arm, the alignment begins by threading the boresight laser beam from the beam-combining table through to a fiducial at the starlight exit of the BRT and then onto the M14 mirror. The dichroic pick-off for the beam-combining table is used to adjust to the BRT, and a fold mirror at the back of the BRT is used to align to M14. A check is made that the boresight laser spot doesn't move by more than $2 \mathrm{~mm}$ at M14 when the FDL carts are exercised front to back.

Alignment through the LDL now begins by checking the alignment on the LDL mirror, M11. A check is made that M14 sends the boresight parallel to the LDL tracks. This is achieved by noting the boresight laser position on M11 with the LDL sled located at front and back of the LDL track. Mirror M14 is adjusted accordingly. The subsequent alignment moves along the beam train, mirror to mirror, aligning each mirror to some target located one mirror up along the beam train. The targets used here can either be targets that are placed over the mirrors that show their geometric centers or the pop-up LED targets themselves. With the LED targets being aligned to prior good alignments along with the increasing confidence of the stability of the LED targets it has become more routine that the LEDs are used as the targets in the process.

Once up at the Nasmyth deck, the alignment of the coudé beam train to the telescope can begin. To do this, measurements of the beam shear and tilt are made, by recording positions of both, before and after $180^{\circ}$ spins of the Keck telescope of that particular interferometer arm. Both measurements should trace semi-circles during the spin and the aim is to adjust the beam train to aim the boresight to the center of rotation of these arcs (i.e., split the difference of the before and after boresight positions). To correct shear, M9 is adjusted; and to correct tilt, M7 is adjusted. This is an iterative procedure that is repeated until there is negligible movement of the boresight laser spot (i.e., converges).

A more precise coudé alignment can now proceed, making use of the AO white light source. The acquisition camera on the AO system is first used to sight both $\mathrm{AO}$ and boresight laser spots. Adjusting M2 brings the interferometer optical axis to be co-aligned with that of the Keck telescope through AO. Further $180^{\circ}$ spins of the Keck telescope are performed, but this time observing the $\mathrm{AO}$ white light source on the angle tracker in the beam-combining room. Again, the difference is split through the adjustment of M7. This is repeated, as necessary, so that that the AO white light source is confined to the central few pixels on the angle tracker with Keck telescope spins.

The coudé alignment is now complete. Most importantly, the light path through the M6 to M7 segment is co-incident with the mechanical azimuthal rotation axis of the Keck telescope.

\subsection{Toward automation}

The scope of the autoalignment system is to automate that part of the alignment that extends from M14 to M4. The alignment of the segment from the beam-combining table to M14 is relatively easy to accomplish and the components are regarded as being relatively stable. This is due, in part, to the inter-component distances being relatively small as well as due to the components being smaller, being in 1" space, as opposed to 4" space. There are no plans to automate this part of the beam train.

For any autoalignment system to work, it needs to be able receive input as what a good alignment is, or at least to be able to measure how far off the beam train is from that alignment. The AACAM has to be able to view the LED targets; that is, they must within the field of view of the camera. With a coudé alignment established, the LED targets can be positioned so that each LED actually lines up with this good alignment. In practice, these fiducials will be at best 
accurate to within a few millimeters, but still this serves as a good starting point for the autoaligner. This set-up step should theoretically be made once.

Another useful input comes from the $850 \mathrm{G}$ motors, as each of these has encoders that can be read to provide a record of mirror positions for the last good alignment. These can be used as an input for the starting point of any subsequent alignment; however, given the length of the beam line and the poor repeatability of regaining a good alignment with past motor positions, at best this is only a starting point

Finally, the LDL track needs to be surveyed. As the angle of the M11 mirror changes for different LDL sled locations along this delay track, the angle of incidence at M9 changes, consequently so too the angle at M7, etc. What may have started as a good coudé alignment is no longer true after an LDL sled move. The purpose of an LDL survey is to provide information as to the orientation of mirrors M9 and M11, so that LED targets to L7 can be observed for all positions along the LDL track. This is an important starting point for an autoalignment to commence.

The LDL is "surveyed" by moving the sled an incremental length along the track and then readjusting mirrors M11 and M9; then recording $850 \mathrm{G}$ motor positions, to build up a lookup table. In this case, the requirement for the survey is that the L9 and L7 targets fall within a nominal 20 pixels of the AACAM center of field of view, AACAM's origin. The functional procedure for generating this is:

- Start with a rough beamtrain alignment such that L7 can be "seen" by AACAM.

- Start with LDL sled at beginning of its track

1. Turn on L7 LED.

2. Take a centroid of L7 position with AACAM. If not at origin, M9 needs adjustment.

3. Move M9, turn off L7, and turn on L9. This occurs simultaneously.

4. Take a centroid of L9 position with AACAM. If not at origin, M11 needs adjustment.

5. Adjust M11, and move M9 to compensate (with appropriate scale factor) so that image of L7 remains stationary. Turn off L9.

- Repeat above six steps until both M9 and M11 require no adjustment.

- Record 850G motor positions values for M11 and M9.

- Move LDL sled an incremental length down the LDL track (e.g., 0.5m).

- Repeat above sequence until end of track is reached.

The end result is a lookup table that allows the LDL system to automatically re-point beam train mirrors during a sled move so that the resulting alignment is ready for the autoaligner to reset the alignment for the new LDL configuration.

\section{THE AUTO-ALIGNER}

The ultimate goal of the autoaligner is to have a system with a single command that would bring the mirrors from M4 to M14 to a sufficiently good coudé alignment, from which it is only necessary to make fine tweaks to the alignment using the angle tracker in the alignment procedure described in Section 2.2. The autoaligner assumes that the alignment from the beam-combining table to M14 is good before continuing, that the LDL sleds have been positioned to their desired positions, and that the M9 and M11 mirrors have been correctly set for that particular location. It also requires that a look-up table be created that contains information on the position of the boresight laser beam with respect to each of the LED targets when the beam train is known to be in a perfect coudé alignment. These are the offset coordinates that are used by the system to adjust the alignment relative to each target.

The basic functional algorithm for the autoaligner is:

- Obtain coordinates of interferometer optical axis with AACAM.

- Insert alignment corner cubes in the beam-combining lab and ensure boresight laser is on.

- Record position of boresight laser with AACAM. This is the position of the optical axis relative to AACAM.

- Remove corner cubes and shutter off boresight laser.

- Turn on L11.

- Capture centroid of L11 
- Adjust M14 until L11's centroid matches the lookup table offset location relative to the interferometer optical axis within a few pixels.

- For each LED target along the beamtrain until L4:

- Turn off presently turned on LED target and turn on the next LED target in beam train in direction of the boresight laser.

- Adjust next actuated mirror down-stream (starlight direction) from LED target to the offset location from lookup table relative to interferometer optical axis.

- $\quad$ Turn off L4.

Note: The telescope azimuth position must be the same during the above procedure as when the LEDs were aligned to the optical axis.

\subsection{Current status}

A fully automated version of the above sequence has not yet been implemented, but many of the pieces for a fully functioning autoaligner have been implemented since the last report on the system ${ }^{1}$. Select features of the software are routinely used to remotely perform alignment tasks, albeit in a manual manner. The LDL tracks have been reliably surveyed so that it is possible to move the LDL sleds and maintain a view of the LED targets throughout the entire length of track. It is possible to obtain centroids and then actuate the $850 \mathrm{G}$ motors. With these pieces the following functionality exits:

- Perform an alignment using LED targets and adjusting mirrors under software control but with human initiation for each mirror in the beamtrain.

- Recovery of coudé alignments after LDL moves, a variant on the overall alignment procedure that concentrates on the M11 and M9 mirrors, but still relies on an operator to initiate mirror adjustments.

In particular, this second function, the recovery of coudé alignments after LDL moves, has been a valuable addition of functionality during interferometer observing runs. It has enabled LDL reconfigurations to be achieved in as little as 15 minutes. This followed with telescope spins that for verification. The overall reconfiguration is completed in a little less than an hour. While this procedure has been executed several times, more work is needed to make it more robust and reliable. As more experience is gained with the reconfiguration procedure, the need for the verification spins should be eliminated.

\section{CONCLUSIONS}

The autoaligner system automates what was previously a manual process to align the interferometer to carry out nightly observations. The design of the system has evolved, especially with respect to the detailed procedures and software, as we have gained experience during its implementation. The results achieved to date are encouraging, and we expect that the full benefits from this system described here will come about from further refinements and use.

\section{ACKNOWLEDGEMENTS}

The research described in this paper was carried out at the W. M. Keck Observatory, and at the Jet Propulsion Laboratory, California Institute of Technology, under a contract with the National Aeronautics and Space Administration. The Keck Interferometer is funded by the National Aeronautics and Space Administration as part of its Navigator Program. The W. M. Keck Observatory is operated as a scientific partnership among the California Institute of Technology, the University of California, and the National Aeronautics and Space Administration. The Observatory was made possible by the generous financial support of the W. M. Keck Foundation.

\section{REFERENCES}

1. P. Wizinowich et al, "Visibility science operations with the Keck Interferometer," in New Frontiers in Stellar Interferometry, SPIE Proc. 5491, 2004.

2. G. van Belle et al, "The Keck Interferometer Autoaligner," in Interferometry for Optical Astronomy II, W. A. Traub, ed., SPIE Proc. 4838, 1246-1255, 2003. 